\title{
Effect of GenF20 Plus on serum IGF-I levels in healthy adults: a randomized controlled study
}

This article was published in the following Dove Press journal:

Open Access Journal of Clinical Trials

25 March 2015

Number of times this article has been viewed

\section{Navneet Sonawane \\ Vinayak Kale ${ }^{2}$ \\ Suhas Erande ${ }^{3}$ \\ Jayesh Chaudhary'}

'Vedic Lifesciences Pvt Ltd, Mumbai, India; ${ }^{2}$ Lokmanya Hospital, Pune, India; ${ }^{3}$ Akshay Hospital, Pune, India
Correspondence: Navneet Sonawane Vedic Lifesciences Pvt Ltd, 203, Morya Landmark I, Off New Link Road, Andheri (West), Mumbai, Maharashtra, India Tel +9l 2242172319

Email navneet_sonawane@yahoo.com
Background: Aging is related to a reduction of growth hormones, resulting in physiological derailment and affects overall wellbeing. GenF20 Plus is a dietary supplement postulated to naturally stimulate the secretion of human growth hormone (HGH) through the anterior pituitary. This study sought to evaluate the effect of GenF20 Plus in enhancing the levels of insulin-like growth factor-1 (IGF-1), which is a marker of HGH levels.

Methods: Seventy subjects aged 35-65 years visiting outpatient departments at five study centers across India, presenting with at least two of the following age-related complaints: decreased memory, decreased libido, low energy levels, or poor quality of sleep were randomly assigned to either GenF20 Plus ( $n=35)$ or placebo $(n=35)$ for a period of 12 weeks ( 84 days). Randomization was carried out using computerized software. The primary outcome measure was serum IGF-1 levels. Changes in waist circumference, body mass index, body fat percentage, lean muscle mass, and scores for memory, libido, energy levels, and quality of sleep were also assessed.

Trial registration: CTRI/2011/06/001784.

Results: Sixty-one subjects completed the study as per protocol and were analyzed. The mean increase (mean \pm standard deviation) in IGF-1 levels at day 84 in the GenF20 Plus group was $13.46 \pm 36.12 \mathrm{ng} / \mathrm{mL}$ and in the placebo group was $6.35 \pm 36.56 \mathrm{ng} / \mathrm{mL}$, which was not statistically significantly different $(P>0.05)$ when compared across the groups. In the $\geq 40$ years subgroup, the mean increase in IGF-1 in the GenF20 Plus group $(14.59 \pm 40.08 \mathrm{ng} / \mathrm{mL})$ was not statistically significantly different when compared to the placebo group $(3.17 \pm 16.09 \mathrm{ng} / \mathrm{mL})$ using analysis of variance (ANOVA; $P>0.05$ ). However, when this change was analyzed using analysis of covariance (ANCOVA) considering baseline values as the covariates, it was found to be statistically significantly different (GenF20 Plus: $22.69 \pm 40.62 \mathrm{ng} / \mathrm{mL}$, placebo: $-4.31 \pm 16.79 \mathrm{ng} / \mathrm{mL}$; $P<0.05)$. The changes in IGF-1 values in the $<40$ years subgroup were not found to be statistically significant. No statistically significant difference over placebo was seen for the other variables.

Conclusion: GenF20 Plus increased serum IGF-1 levels in subjects $\geq 40$ years of age who physiologically have reduced IGF-1 levels. However, significant changes in other parameters were not seen, probably due to the short duration of treatment. Further studies with longer treatment duration and large sample size are warranted to understand the clinical benefits of GenF20 Plus, especially in adults above 40 years of age.

Keywords: IGF-1, herbal supplement, human growth hormone, HGH, aging

\section{Introduction}

Aging is a phenomenon related to a series of catabolic processes resulting in decreased function and structural integrity of several physiological systems. These changes in 
the aging phenotype are correlated with a decline in growth hormone secretion and a resulting decrease in plasma levels of its anabolic mediator, insulin-like growth factor-1 (IGF-1). ${ }^{1,2}$

Human growth hormone $(\mathrm{HGH})$ is a naturally occurring peptide hormone secreted by the pituitary gland. ${ }^{3}$ It is secreted in a pulsatile fashion, generally following a circadian rhythm. HGH has many varied roles throughout life, from growth itself, including the turnover of muscle, bone, and collagen, to the regulation of selective aspects of metabolic function, including increased fat metabolism and the maintenance of a healthier body composition in later life. ${ }^{4,5}$ Secretion of growth hormone decreases with increasing age. ${ }^{6}$ There is a physiological decline in HGH and IGF-1 levels as age increases, resulting in disturbed sleep cycles and adversely affecting the memory of an individual. ${ }^{7,8}$ Studies have shown improvement in cognitive functioning in $\mathrm{HGH}$-deficient patients by $\mathrm{HGH}$ substitution. ${ }^{9}$ Thus, restoring HGH levels may stimulate sound sleep and improve memory and the overall quality of life.

The current available therapy is targeted for $\mathrm{HGH}$ replacement only in $\mathrm{HGH}$-deficient individuals, but not for healthy individuals or age-related physiological decline of $\mathrm{HGH}$. HGH and IGF-1 are also used as performanceenhancing agents, to increase muscle mass and exercise endurance. ${ }^{10}$ Given its potentially adverse effects like carcinogenic activity, ${ }^{11}$ administration of exogenous $\mathrm{HGH}$ and IGF-1 is not a safe method. The growing abuse of $\mathrm{HGH}$ for muscle building by athletes and body builders and its related medicolegal issues also necessitates finding a safe and acceptable alternative. Many herbs and amino acids are known to combat aging, boost bodily functions, improve physical stamina, increase lean muscle mass, improve libido, memory, and quality of sleep. ${ }^{12-14}$ GenF20 Plus is a dietary supplement postulated to naturally stimulate the secretion of HGH through the anterior pituitary. It intends to provide a solution to improve IGF-1 levels and in turn, HGH levels without any adverse effects.

The objective of this study was to assess the efficacy of GenF20 Plus in increasing the levels of IGF-1 and its related effects on memory, libido, energy levels, and quality of sleep.

\section{Methods}

The study was reviewed and approved by the Independent Ethics Committee - Aditya, Ahmedabad, Gujarat, India; and conducted according to the principles of good clinical research practice and the Declaration of Helsinki. Voluntary, written informed consent was obtained from all subjects prior to initiation of any study-related procedure.

\section{Study design}

A double-blind placebo-controlled design was chosen for the study. The subjects were assessed for the recruitment criteria during the screening visit and then randomized in the ratio 1:1 at Day 0 to receive either GenF20 Plus tablets and liquid or placebo tablets and liquid. The follow-up visits were at an interval of 4 weeks each (Day 28, Day 56, and Day 84).

\section{Investigational products}

GenF20 Plus is a dietary supplement consisting of tablets containing L-glutamine, L-arginine $\mathrm{HCl}$, L-glycine, L-tyrosine, Tribulus terrestris extract, L-lysine $\mathrm{HCl}$, astragalus root, colostrum powder, deer velvet antler powder, gamma-amino butyric acid (GABA), L-isoleucine, anterior pituitary powder, phosphatidylcholine, L-valine, L-ornithine, glucose-tolerance factor (GTF), and chromium, and liquid containing alpha glycerylphosphorylcholine (GPC), GABA, Mucuna pruriens, moomiyo extract, ornithine alpha ketoglutarate, L-glutamine, L-arginine, L-lysine, L-valine, L-isoleucine, L-tyrosine, and glycine with a palatable berry flavor. Matching placebo tablets were made up of carboxymethyl cellulose tablets and liquid was made up of berry-flavored distilled water with sodium saccharin and sucralose.

The subjects were asked to take two tablets orally with water and $2 \mathrm{~mL}$ of liquid sublingually before meals in the morning and evening.

\section{Randomization and blinding}

The master randomization chart with blocks of four was generated using statistical software (Stats Direct v 2.7.8; Stats Direct Ltd, Altrincham, UK). At Day 0, subjects were randomized to receive either GenF20 Plus or placebo by the study coordinator based on the next available number as per the randomization chart. The tablets of GenF20 Plus and placebo were identical with respect to size, average weight, thickness, and color and packed in identical bottles. GenF20 Plus and the placebo liquid were identical in terms of color, flavor, and viscosity and were packed in identical plastic amber-colored bottles.

Each subject was issued study medications in pre-labeled identical cartons (containing a bottle of 124 tablets and three bottles of liquid) at each visit. The labeling and masking was done at the manufacturing unit, under the supervision of quality assurance personnel. The blinding codes were maintained in the site master files in sealed, tamper-evident envelopes and were checked by the study monitor during each monitoring visit. 


\section{Subjects}

Potential subjects were screened from patients attending outpatient departments at five study centers across India from July 2011 to January 2012.

Male and female subjects aged 35-65 years with body mass index (BMI) between $18.5-29.9 \mathrm{~kg} / \mathrm{m}^{2}$ having any two of the following age-related conditions: decreased memory, decreased libido, low energy levels, or poor quality of sleep, with grade 1 or 2 on a five-point Likert scale (1: poor, 2: fair, 3: good, 4: very good, 5: excellent) for at least 1 month but not for more than 6 months were offered participation in the study. Other inclusion criteria were willingness to give written informed consent and abide with study procedures, come for follow-up visits, and continue current lifestyle practices with no modifications (in diet and exercise) during the study period. Women who were willing to practice suitable contraception (except oral contraceptives) during the study were recruited.

The exclusion criteria were subjects with uncontrolled diabetes, hypertension, hypothyroidism and hyperthyroidism, hepatic or renal impairment, acromegaly, significant cardiovascular comorbidities, and debilitating neurological or psychiatric disorders including seizure disorders, depression, and drugs used in these conditions. Pregnant and lactating women, women on oral contraceptives, estrogen supplements, or corticosteroids, who had known cases of estrogen-sensitive disorders such as breast cancer, uterine cancer, ovarian cancer, endometriosis, and uterine fibroids were also excluded. Subjects with hypersensitivity or allergy to one or more of the ingredients of the investigational product, allergy to milk and milk products, or those who had recently ( $<1$ month) participated in a clinical trial were excluded. Heavy smokers $(>10$ cigarettes per day) and chronic alcoholics were also excluded. Subjects who had taken any drugs or supplements in the preceding 1 month for decreasing body fat, increasing libido and energy levels, and improving quality of sleep and memory, or those who had started an exercise and/or diet regimen within 30 days of the screening visit were excluded.

\section{Study procedures}

The subjects were called for a screening visit 5 days before randomization to assess their eligibility for the study. Blood and urine samples were collected to assess laboratory parameters (complete blood count $[\mathrm{CBC}]$, urine routine, glycated hemoglobin [HbA1c], serum glutamate pyruvate transaminase [SGPT], serum creatinine, triiodothyronine [T3], tetraiodothyronine [T4], thyroid-stimulating hormone $[\mathrm{TSH}])$ and screen out patients with related exclusion criteria.
An electrocardiogram (ECG) was undertaken and urine pregnancy test was performed to rule out pregnancy.

The eligible subjects were randomized at Day 0 and called for follow-up visits on Day 28, Day 56, and Day 84. At each visit, complete physical examination was undertaken and waist circumference (WC) was recorded. Also, subjects were asked to rate their feelings or responses for the parameters of memory, libido, energy levels, and quality of sleep on a scale of 1-5 (1: poor, 2: fair, 3: good, 4: very good, 5: excellent). BMI, body fat percentage, and lean body mass were measured using Omron body fat analyzer HBF-200. Blood samples were collected after an overnight fast of 10-12 hours for assessment of serum IGF-1 levels at Day 0 and Day 84 .

At Day 84, blood and urine assessments were performed to assess the safety profile and ECG was recorded. Global assessment of efficacy by subject was rated as good, fair, and poor based on their perception of their improvement in overall health.

\section{Monitoring and quality control}

Regular monitoring visits and independent audit visits were carried out at each site to ensure data quality and compliance to protocol. The investigator informed the subjects of the study procedures and their obligations and responsibilities during the study. At each visit, the record of dispensed and returned investigational product was maintained in order to ensure that the subject was taking the investigational product properly throughout the treatment duration.

All the laboratory biochemical tests including serum IGF-1 levels were evaluated at a central laboratory - Metropolis Healthcare Pvt Ltd, Mumbai, India.

\section{Statistics}

Since this was the first exploratory clinical study on GenF20 Plus, no statistical method was used for sample size calculation. An arbitrarily chosen sample size of 60 completed subjects, with 30 in each group, was planned in the study to detect a statistical difference between GenF20 Plus and placebo. Considering a withdrawal and dropout rate of $25 \%$, we planned to recruit a maximum of 80 subjects in the study.

All statistical tests were applied with $95 \%$ confidence intervals and performed using Epi Info 3.5.1 and Microsoft Excel 2007. The efficacy analyses were conducted on the per protocol population. Mean changes in baseline characteristics in terms of vital parameters, laboratory hematological tests, BMI, body fat percentage, lean body mass, WC, and parameters of memory, libido, energy levels, and quality of sleep from Day 0 to Day 84 were compared across the two 
groups using analysis of variance (ANOVA). Changes in IGF-1 values from Day 0 to Day 84 were compared across the two groups using ANOVA. A subgroup analysis by ANOVA was done post hoc, with two subgroups based on the subjects' age; group 1 with subjects aged $\geq 40$ years and group 2 with subjects aged $<40$ years. As the mean ages for the GenF20 Plus and placebo groups were 40.57 and 40 years, respectively, and median 38 and 39 years, respectively, 40 years was selected as the cut-off for the subgroups. One-way analysis of covariance (ANCOVA) was then performed to adjust the baseline variations for IGF-1.

For global assessment, good and fair was clubbed together as a satisfactory response and poor was denoted as an unsatisfactory response and was evaluated using Pearson's chi-square test.

Safety was evaluated by comparing the number of adverse events in both the treatment groups and assessing changes in vital parameters, hematology, and biochemistry parameters.

\section{Results}

\section{Disposition of subjects}

The details of the subjects recruited and completing the study are presented in Figure 1. Ninety-eight subjects were screened for the study; out of which 28 were screening failures. The remaining 70 subjects were randomized and allocated to either the GenF20 Plus $(n=35)$ or placebo $(n=35)$ groups.

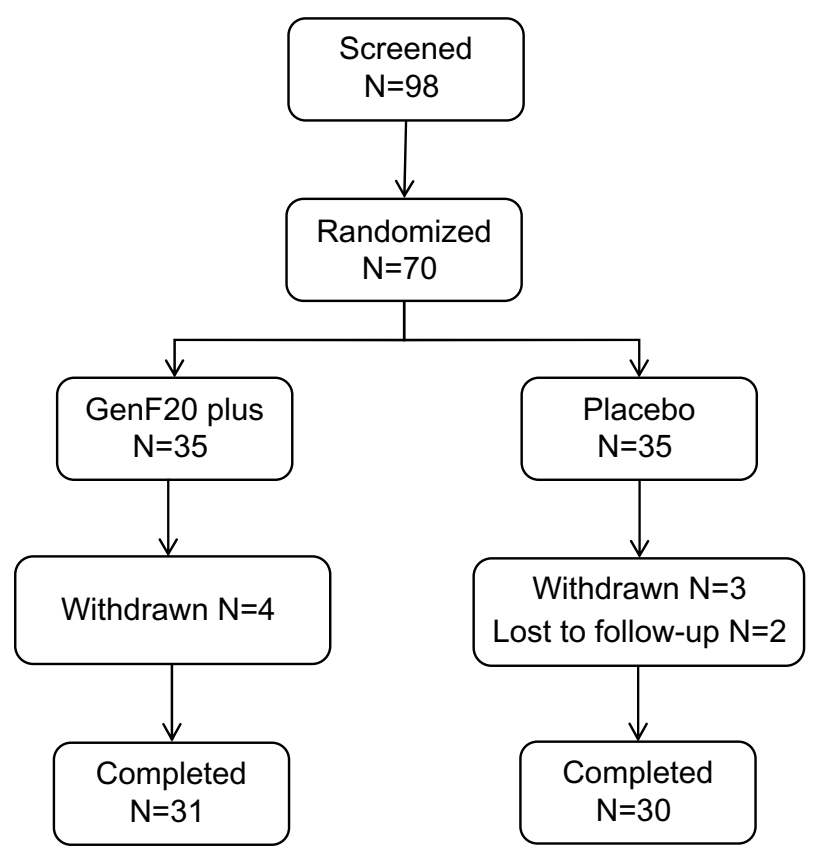

Figure I Disposition of subjects.
The major reasons for screening failures included high HbA1c levels $(n=7)$, abnormal TSH levels $(n=6)$, low platelet count $(n=2)$, and high BMI $(n=2)$. Eight subjects were unwilling to participate in the study. Other reasons for screening failure were presence of calcium oxalate crystals in urine $(n=1)$, elevated serum creatinine $(n=1)$, and abnormal leukocyte count $(n=1)$.

Out of the 70 randomized subjects, seven subjects were withdrawn from the study because they were unwilling to undergo laboratory tests and two subjects were lost to follow-up. A total of 61 subjects completed the study; GenF20 Plus $(n=31)$ and placebo $(n=30)$.

\section{Baseline characteristics}

The demographics and key baseline characteristics for the subjects recruited in the study are presented in Table 1 . There was no statistically significant difference between the two groups at baseline in terms of demographics, BMI, IGF-1 levels, and duration of complaints; indicating effectiveness of randomization.

\section{Serum IGF-I levels}

The changes in serum IGF-1 levels are presented in Table 2. There was an increase seen in IGF-1 values from baseline to the end of treatment in both the groups; however, it failed to reach statistical significance. This change was also

Table I Demographic data and baseline characteristics

\begin{tabular}{lll}
\hline Baseline characteristics & $\begin{array}{l}\text { GenF20 Plus } \\
(\mathbf{n = 3 5 )}\end{array}$ & $\begin{array}{l}\text { Placebo } \\
(\mathbf{n}=35)\end{array}$ \\
\hline $\begin{array}{l}\text { Age (years) } \\
\text { - Mean (SD) }\end{array}$ & $40.57(6.0 \mathrm{I})$ & $42.00(7.2 \mathrm{I})$ \\
Sex & $18(5 \mathrm{I} .43)$ & $13(37.14)$ \\
- Male N (\%) & $17(48.57)$ & $22(62.86)$ \\
- Female N (\%) & $35(100)$ & $3 \mathrm{I}(88.57)$ \\
Low energy N (\%) & $18(5 \mathrm{I} .43)$ & $13(37.14)$ \\
$\begin{array}{l}\text { Decreased memory N (\%) } \\
\text { Decreased libido N (\%) }\end{array}$ & $9(25.7 \mathrm{I})$ & $10(28.57)$ \\
$\begin{array}{l}\text { Poor quality of sleep N (\%) } \\
\text { Serum IGF-I levels (ng/mL) }\end{array}$ & $27(77.14)$ & $32(91.43)$ \\
- Mean (SD) & $134.52(44.17)$ & $123.47(44.59)$ \\
$\begin{array}{l}\left.\text { BMI (kg/m }{ }^{2}\right) \\
\text { - Mean (SD) }\end{array}$ & $24.59(3.15)$ & $25.47(2.94)$ \\
$\begin{array}{l}\text { BMI category } \\
\text { - Normal N (\%) }\end{array}$ & $19(54.29)$ & $13(37.14)$ \\
- Overweight N (\%) & $16(45.7 \mathrm{I})$ & $2 \mathrm{I}(60)$ \\
- Obese N (\%) & $0(0)$ & $\mathrm{I}(2.86)$ \\
$\begin{array}{l}\text { Duration of complaints (days) } \\
\text { - Mean (SD) }\end{array}$ & $89.57(39.16)$ & $83.5 \mathrm{I}(37.22)$ \\
\hline
\end{tabular}

Abbreviations: BMI, body mass index; IGF-I, insulin-like growth factor-I; SD, standard deviation. 
Table 2 Changes in serum insulin-like growth factor $(\mathrm{ng} / \mathrm{mL})$

\begin{tabular}{|c|c|c|c|c|c|c|}
\hline \multirow{3}{*}{$\begin{array}{l}\text { Group } \\
\text { All } \\
\text { subjects }\end{array}$} & \multicolumn{3}{|c|}{ GenF20 Plus } & \multicolumn{3}{|c|}{ Placebo } \\
\hline & Day 0 & Day 84 & Change & Day 0 & Day 84 & Change \\
\hline & \multicolumn{3}{|l|}{$\mathbf{N}=3 \mathbf{I}$} & \multicolumn{3}{|l|}{$\mathbf{N}=\mathbf{3 0}$} \\
\hline $\begin{array}{l}\text { Mean } \\
(\mathrm{ng} / \mathrm{mL})\end{array}$ & 134.52 & 147.98 & 13.46 & 123.47 & $|29.8|$ & 6.35 \\
\hline SD & 44.17 & 45.19 & 36.12 & 44.59 & 52.13 & 36.56 \\
\hline Min & 56.14 & 51.1 & -46 & 40.8 & 60.5 & -43 \\
\hline Max & 224 & 277 & 86 & 243 & 247 & II5.I \\
\hline
\end{tabular}

Abbreviation: SD, standard deviation.

not statistically significant $(P>0.05)$ when compared across the groups.

In the subgroup analyses (Table 3 ), there was an increase seen in the IGF-1 values from Day 0 to Day 84 in the four groups which was not statistically significant using ANOVA. Mean serum IGF-1 levels at Day 0 in the subgroups was found to be significantly different for the GenF20 Plus and placebo groups; hence, the mean change in IGF-1 values was analyzed by ANCOVA, taking the IGF-1 values at Day 0 as the covariate. The change was found to be statistically significant $(P<0.05)$ for the $\geq 40$ years subgroup when compared across the treatment groups and not statistically significant $(P>0.05)$ for the $<40$ years age group.

\section{Waist circumference, BMI, body fat percentage, and lean body mass}

There was a reduction seen in WC, BMI, and body fat percentage in both GenF20 Plus and placebo groups at Day 84; however, it was not statistically significant $(P>0.05)$ when compared within groups and across groups. The lean body mass increased slightly in both the groups but did not reach statistical significance (Table 4).

\section{Memory, libido, energy levels, and quality of sleep}

There was improvement in all the variables from baseline to the end of treatment in both GenF20 Plus and placebo groups. The change was statistically significant for energy levels and sleep quality in both the GenF20 Plus and placebo groups and for memory in the placebo group from Day 0 to Day 84, but was not statistically significant across the two groups (Table 5).

\section{Global assessment by subjects}

Twenty-one (67.74\%) and 18 (60\%) subjects rated the overall improvement as satisfactory and ten $(32.26 \%)$ and $12(40 \%)$ rated it as unsatisfactory in the GenF20 Plus and placebo groups, respectively. However, this was not statistically significant $(P<0.05)$ as shown by Pearson's chi-square test.

\section{Safety}

Twelve adverse events occurred in the entire study (seven in the GenF20 Plus group and five in the placebo group); ten of which were of the gastrointestinal system (eight hyperacidity cases and two cases with pain in the abdomen). The other two events were headache and skin eruptions just below the eyes. Except for the two pain in the abdomen events, which were of moderate severity, the events were mild in severity. All adverse events were judged as "probably related" or "not related" to the study products. All events were completely resolved before the end of the study.

There were no clinically or statistically significant changes seen in pulse rate, blood pressure, and temperature.

Changes in laboratory measurements were analyzed in a subset of 52 subjects from per protocol population for whom complete laboratory data was available. When compared for changes from screening to Day 84, no statistically

Table 3 Changes in serum insulin-like growth factor for subgroups $(\mathrm{ng} / \mathrm{mL})$

\begin{tabular}{|c|c|c|c|c|c|c|c|c|}
\hline & \multicolumn{4}{|c|}{ GenF20 Plus } & \multicolumn{4}{|c|}{ Placebo } \\
\hline & Day 0 & Day 84 & $\begin{array}{l}\text { Observed } \\
\text { change }\end{array}$ & $\begin{array}{l}\text { Adjusted } \\
\text { change }\end{array}$ & Day 0 & Day 84 & $\begin{array}{l}\text { Observed } \\
\text { change }\end{array}$ & $\begin{array}{l}\text { Adjusted } \\
\text { change }\end{array}$ \\
\hline Age $\geq 40$ years & $\mathbf{N}=13$ & & & & $\mathbf{N}=\mid \mathbf{3}$ & & & \\
\hline Mean (ng/mL) & $|30.6|$ & $\mid 45.20$ & 14.59 & $22.69^{a}$ & 96.67 & 99.84 & 3.17 & -4.31 \\
\hline SD & 47.08 & 36.55 & 40.08 & 40.62 & 29.54 & 27.30 & 16.09 & 16.79 \\
\hline Min & 61.8 & 89.8 & -46 & & 40.8 & 65.8 & -25.5 & \\
\hline Max & 217 & 206 & 86 & & 114 & 160 & 42.8 & \\
\hline Age $<40$ years & $\mathbf{N}=18$ & & & & $\mathbf{N}=17$ & & & \\
\hline Mean (ng/mL) & 138.13 & |49.84 & $12.7 \mid$ & 12.06 & 143.96 & 152.73 & 8.78 & 9.46 \\
\hline SD & 42.31 & 51.09 & 34.41 & 37.41 & 43.82 & 55.48 & 47.05 & 47.92 \\
\hline Min & 56.4 & 51.1 & -30 & & 74.3 & 60.5 & -43 & \\
\hline Max & 224 & 277 & 84 & & 243 & 247 & II5.I & \\
\hline
\end{tabular}

Note: a'Statistically significant using ANCOVA.

Abbreviations: ANCOVA, analysis of covariance; SD, standard deviation. 
Table 4 Changes in waist circumference, BMI, body fat percentage, and lean body mass

\begin{tabular}{|c|c|c|c|c|c|c|}
\hline & \multicolumn{3}{|c|}{ GenF20 Plus (N=3I) } & \multicolumn{3}{|c|}{ Placebo $(\mathbf{N}=30)$} \\
\hline & Day 0 & Day 84 & Change & Day 0 & Day 84 & Change \\
\hline Waist circumference $(\mathrm{cm})$ & $36.26(5.04)$ & $35.96(4.85)$ & $-0.30(0.8 \mathrm{I})$ & $36.12(5.19)$ & $35.87(5.01)$ & $-0.25(0.76)$ \\
\hline BMI $\left(\mathrm{kg} / \mathrm{m}^{2}\right)$ & $25.78(8.27)$ & $25.67(8.23)$ & $-0.11(0.63)$ & $25.43(2.88)$ & $25.23(2.95)$ & $-0.20(0.79)$ \\
\hline Body fat percentage (\%) & $26.55(10.97)$ & $25.66(11.65)$ & $-0.89(1.85)$ & $28.16(7.06)$ & $27.75(7.35)$ & $-0.4 \mathrm{I}(\mathrm{I} .34)$ \\
\hline Lean body mass (kg) & 47.31 (6.92) & $47.59(6.99)$ & $0.27(0.88)$ & $47.4 I(7.59)$ & $47.53(7.79)$ & $0.12(0.99)$ \\
\hline
\end{tabular}

Note: Values are expressed as mean (SD).

Abbreviations: BMI, body mass index; SD, standard deviation.

significant changes were observed in the hematology and biochemical laboratory variables within each group and between the two groups except for an increase in neutrophil percentage in the GenF20 Plus group $(57.56 \% \pm 7.47 \%$ to $62.04 \% \pm 6.69 \%$; mean \pm standard deviation $[\mathrm{SD}]$ ) and a reduction in serum creatinine levels in the placebo group ( $0.86 \pm 0.19$ to $0.77 \pm 0.15 \mathrm{mg} / \mathrm{dL}$; mean $\pm \mathrm{SD}$ ). However, this change was not clinically significant.

\section{Discussion}

Serum IGF-1 and HGH levels are known to decrease with increasing age and are related to the physiological changes in lean body mass, ${ }^{15}$ sleep, ${ }^{7}$ memory, ${ }^{8}$ and energy levels ${ }^{16}$ that are due to increasing age. Replenishing depleting serum IGF-1 and HGH levels is postulated to provide the benefits of younger age and relieve the signs and symptoms of growth hormone decline. A clinical study by Rudman et al has shown beneficial effects of $\mathrm{HGH}$ administration in a group of elderly healthy men with low plasma IGF-I values, but no underlying pituitary pathology. ${ }^{17}$ Sattler et al also demonstrated that HGH supplementation increased lean mass, muscle strength, and aerobic endurance with significant reductions in whole-body and trunk fat. ${ }^{18}$ These studies support the hypothesis that increased levels of IGF-1 and HGH will stimulate lipolysis and cause a reduction in body fat. An improvement in cognitive functioning in HGH-deficient patients by HGH substitution has also been shown in a few studies..$^{19,20}$
The present study was undertaken with a postulated role of GenF20 Plus in stimulating the anterior pituitary gland to secrete $\mathrm{HGH}$, which when released into the blood stream, stimulates the liver to produce IGF-1, the primary mediator of the effects of HGH. GenF20 Plus contains essential amino acids and other ingredients that are known to stimulate the production and secretion of $\mathrm{HGH}$ from the anterior pituitary gland. Amino acids are known to improve HGH levels. ${ }^{21}$ Arwert et al showed that a supplement containing glycine, glutamine, and niacin increased serum HGH levels by $70 \%$ relative to placebo and individual increases in IGF-1 were associated with improved memory and vigor. ${ }^{22}$

In the present study, from baseline to the end of treatment, serum IGF-1 levels increased more in the GenF20 Plus group than in the placebo group. However, this increase was neither clinically nor statistically significant. In the subgroup analysis based on age using ANCOVA, in the $\geq 40$ years subgroup, adjusted percentage change in IGF- 1 was $28.57 \%$ in the GenF20 Plus group as compared to $-0.55 \%$ in the placebo group, a difference that was statistically significant $(P<0.05)$. In the $<40$ years subgroup, there was no marked change in either of the treatment groups. This could be attributed to the sustained inherent mechanism of the body to be able to secrete normal levels of serum IGF-1 levels below 40 years of age. This analysis enables us to postulate that GenF20 Plus is able to stimulate secretion of HGH and IGF-1; this change is noticeably observed in those above 40 years of age.

Table 5 Effect on memory, libido, energy levels, and quality of sleep

\begin{tabular}{|c|c|c|c|c|c|c|}
\hline Variables & Day 0 & Day 84 & Change & Day 0 & Day 84 & Change \\
\hline Memory & \multicolumn{3}{|c|}{ GenF20 Plus $(\mathbf{N}=22)$} & \multicolumn{3}{|c|}{ Placebo (N=19) } \\
\hline $\mathrm{N}=4 \mathrm{I}$ & $2.04(0.90)$ & $2.76(1.16)$ & $0.4 I(0.80)$ & $2.10(0.81)$ & $2.77(1.15)^{\mathrm{a}}$ & $0.3 \mathrm{I}(0.48)$ \\
\hline Libido & \multicolumn{3}{|c|}{ GenF20 Plus $(\mathrm{N}=\mid 8)$} & \multicolumn{3}{|c|}{ Placebo $(\mathrm{N}=15)$} \\
\hline$N=33$ & $2.50(0.78)$ & $3.00(1.14)$ & $0.17(0.7 I)$ & $2.47(0.99)$ & $3.05(1.35)$ & $0.20(0.41)$ \\
\hline Energy levels & \multicolumn{3}{|c|}{ GenF20 Plus $(\mathrm{N}=3 \mathrm{I})$} & \multicolumn{3}{|c|}{ Placebo $(\mathrm{N}=\mathbf{2 8})$} \\
\hline $\mathrm{N}=59$ & $1.16(0.37)$ & $2.39(0.80)^{\mathrm{a}}$ & $1.22(0.80)$ & $\mathrm{I} .2 \mathrm{I}(0.50)$ & $2.14(0.93)^{\mathrm{a}}$ & $0.93(0.86)$ \\
\hline Quality of sleep & \multicolumn{3}{|c|}{ GenF20 Plus $(\mathrm{N}=27)$} & \multicolumn{3}{|c|}{ Placebo $(\mathrm{N}=29)$} \\
\hline$N=56$ & $1.52(0.70)$ & $2.55(0.93)^{\mathrm{a}}$ & $\mathrm{I} .04(0.85)$ & $1.24(0.63)$ & $2.10(1.08)^{\mathrm{a}}$ & $0.86(1.02)$ \\
\hline
\end{tabular}

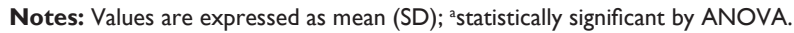

Abbreviations: ANOVA, analysis of variance; SD, standard deviation. 
At the end of 12 weeks of treatment, BMI, WC, body fat, and lean body mass did not show a significant change from baseline to the end of treatment in both the GenF20 Plus and placebo groups. There was a statistically significant improvement in memory, energy levels, and quality of sleep from baseline to the end of treatment in both the groups $(P<0.05)$, but it failed to achieve statistical significance when compared between the two groups. No statistically significant difference was seen between the two groups for global assessment by subjects when assessed by Pearson's chi-square test.

GenF20 Plus was well-tolerated by all the subjects. There were a total of 12 adverse events reported (seven in the active group and five in the placebo group) during the study. They were mild, not related to the study products, and were successfully resolved. No serious adverse events occurred in the study. The present study has not shown improvement in all the parameters assessed, possibly due to the short duration of the study. It failed to show considerable reduction in BMI, WC, or body fat in both the GenF20 Plus and placebo groups. The parameters of sleep, memory, libido, and energy levels also did not show substantial improvement as compared to placebo. Improvement in all of the above parameters is difficult to attain in a short duration of 12 weeks. The longer the duration of these impairments, the longer the time required to attain normalcy or perceivable benefits by any agent. The fact that serum IGF-1 levels increased with statistical significance in the $\geq 40$ years subgroup is an indication that prolonged usage of GenF20 Plus may show improvement in other parameters as well. Prolonged consumption of GenF20 Plus should increase HGH and IGF-1 levels and manifest improvement in the quality of life parameters. GenF20 Plus may be required to be consumed for an extended period of time to show any considerable improvement in weight and body fat.

\section{Conclusion}

This was the first study done on GenF20 Plus to assess its effects on IGF-1 levels. Prolonged usage of GenF20 Plus may be able to show a clinically significant increase in serum IGF-1 levels. In summary, GenF20 Plus may not have delivered the projected efficacy results in this study, but is certainly worthy of further exploration as a potential agent to improve quality of life in the overweight and aging population.

\section{Acknowledgments}

We acknowledge the support provided by Aliya Shakeel in the concept and design of the study and Arun Nanivadekar for his guidance and incessant support. We also acknowledge the contribution of Pravin Supe, Rahul Patil, and Shivram
Bhonagiri in the collection of patient data and the financial support received from DM Contact Management for the study. The complete Clinical Study Report is available at http:// www.genf20-plus.com/GenF20Plus-Study-19-7-2012.pdf?

\section{Author contributions}

Navneet Sonawane was involved with the conception and design of the study, analysis and interpretation of data, and drafted the manuscript. Suhas Erande and Vinayak Kale collected patient data and reviewed the manuscript. Jayesh Chaudhary participated in the design of the study and provided expert comments on the manuscript. All authors critically reviewed and approved the manuscript and are accountable for the entire study.

\section{Disclosure}

This study was conducted by Vedic Lifesciences Ltd with financial support from DM Contact Management. Vedic Lifesciences Ltd is an independent research organization, which is in no way related to DM Contact Management nor has any financial interests in the results of the study. The authors did not have any financial or commercial competing interests to declare in relation to this manuscript.

\section{References}

1. Khan AS, Sane DC, Wannenburg T, Sonntag WE. Growth hormone, insulin-like growth factor-1 and the aging cardiovascular system. Cardiovasc Res. 2002;54(1):25-35.

2. Giovannini S, Marzetti E, Borst SE, Leeuwenburgh C. Modulation of GH/IGF-1 axis: potential strategies to counteract sarcopenia in older adults. Mech Ageing Dev. 2008;129(10):593-601.

3. Saugy M, Robinson N, Saudan C, Baume N, Avois L, Mangin P. Human growth hormone doping in sports. Br J Sports Med. 2006;40 Suppl 1: i35-i39.

4. Godfrey RJ, Madgwick Z, Whyte GP. The exercise-induced growth hormone response in athletes. Sports Med. 2003;33(8):599-613.

5. Ho KY, Veldhuis JD, Johnson ML, et al. Fasting enhances growth hormone secretion and amplifies the complex rhythms of growth hormone secretion in man. J Clin Invest. 1988;81(4):968-975.

6. Juul A, Main K, Blum WF, Lindholm J, Ranke MB, Skakkebaek NE. The ratio between serum levels of insulin like growth factor (IGF)-1 and the IGF binding proteins (IGFBP-1, 2 and 3) decreases with age in healthy adults and it is increased in acromegalic patients. Clin Endocrinol (Oxf). 1994;41(1):85-93.

7. Vgontzas AN, Tsigos C, Bixler EO, et al. Chronic insomnia and activity of the stress system: a preliminary study. J Psychosom Res. 1998;45(1): 21-31.

8. Zelinski EM, Burnight KP. Sixteen-year longitudinal and time lag changes in memory and cognition in older adults. Psychol Aging. 1997;12(3):503-513.

9. Schneider HJ, Pagotto U, Stalla GK. Central effects of the somatotropic system. Eur J Endocrinol. 2003;149(5):377-392.

10. Velloso CP. Regulation of muscle mass by growth hormone and IGF-I. Br J Pharmacol. 2008;154(3):557-568.

11. Tentori L, Graziani G. Doping with growth hormone/IGF-1, anabolic steroids or erythropoietin: is there a cancer risk? Pharmacol Res. 2007; 55(5):359-369. 
12. Alba-Roth J, Müller OA, Schopohl J, von Werder K. Arginine stimulates growth hormone secretion by suppressing endogenous somatostatin secretion. J Clin Endocrinol Metab. 1988;67(6):1186-1189.

13. Neuzillet Y, Hupertan V, Cour F, Botto H, Lebret T. A randomized, double-blind, crossover, placebo-controlled comparative clinical trial of arginine aspartate plus adenosine monophosphate for the intermittent treatment of male erectile dysfunction. Andrology. 2013;1(2): 223-228.

14. Fukagawa NK. Protein and amino acid supplementation in older humans. Amino Acids. 2013;44(6):1493-1509.

15. Veldhuis JD, Iranmanesh A, Ho KK, Waters MJ, Johnson ML, Lizarralde G. Dual defects in pulsatile growth hormone secretion and clearance subserve the hyposomatotropism of obesity in man. J Clin Endocrinol Metab. 1991;72(1):51-59.

16. Clark MR, Katon W, Russo J, Kith P, Sintay M, Buchwald D. Chronic fatigue: risk factors for symptom persistence in a 2 1/2-year follow-up study. Am J Med. 1995;98(2):187-195.

17. Rudman D, Feller AG, Nagraj HS, et al. Effects of human growth hormone in men over 60 years old. N Engl J Med. 1990;323(1):1-6.
18. Sattler FR, Castaneda-Sceppa C, Binder EF, et al. Testosterone and growth hormone improve body composition and muscle performance in older men. J Clin Endocrinol Metab. 2009;94(6):1991-2001.

19. van Dam PS, Aleman A, de Vries WR, et al. Growth hormone, insulinlike growth factor I and cognitive function in adults. Growth Horm IGF Res. 2000;10 Suppl B:S69-S73.

20. Baker LD, Barsness SM, Borson S, et al. Effects of growth hormonereleasing hormone on cognitive function in adults with mild cognitive impairment and healthy older adults: results of a controlled trial. Arch Neurol. 2012;69(11):1420-1429.

21. Zajac A, Poprzecki S, Zebrowska A, Chalimoniuk M, Langfort J. Arginine and ornithine supplementation increases growth hormone and insulin-like growth factor- 1 serum levels after heavy-resistance exercise in strength-trained athletes. J Strength Cond Res. 2010;24(4): 1082-1090.

22. Arwert LI, Deijen JB, Drent ML. Effects of an oral mixture containing glycine, glutamine and niacin on memory, GH and IGF-I secretion in middle-aged and elderly subjects. Nutr Neurosci. 2003;6(5):269-275.
Open Access Journal of Clinical Trials

\section{Publish your work in this journal}

The Open Access Journal of Clinical Trials is an international, peerreviewed, open access journal publishing original research, reports, editorials, reviews and commentaries on all aspects of clinical trial design, management, legal, ethical and regulatory issues, case record form design, data collection, quality assurance and data auditing

\section{Dovepress}

methodologies. The manuscript management system is completely online and includes a very quick and fair peer-review system, which is all easy to use. Visit http://www.dovepress.com/testimonials.php to read real quotes from published authors. 